\title{
Characterization and Application of a Grazing Angle Objective for Quantitative Infrared Reflection Microspectroscopy
}

\author{
STEPHEN V. PEPPER \\ National Acronawtics and Space Administration, Lewis Research Center, Cleveland. Ohio 44135
}

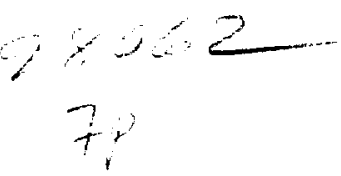

\begin{abstract}
A grazing angle objective on an infrared microspectrometer is studied for quantitative spectroscopy by considering the angular dependence of the incident intensity within the objective's angular aperture. The assumption that there is no angular dependence is tested by comparing the experimental reflectance of $\mathrm{Si}$ and $\mathrm{KBr}$ surfaces with the reflectance calculated by integrating the Fresnel reflection coefficient over the angular aperture under this assumption. Good agreement was found, indicating that the specular reflectance of surfaces can straightforwardly be quantitatively integrated over the angular aperture without considering nonuniform incident intensity. This quantitative approach is applied to the thickness determination of dipcoated Krytox on gold. The infrared optical constants of both materials are known, allowing the integration to be carried out. The thickness obtained is in fair agreement with the value determined by ellipsometry in the visible. Therefore, this paper illustrates a method for more quantitative use of a grazing angle objective for infrared reflectance microspectroscopy.

Index Headings: Infrared; Microspectroscopy; Thin films.
\end{abstract}

\section{INTRODUCTION}

Infrared microspectroscopy is widely used for vibrational chemical analysis of materials at lateral resolutions as small as ten micrometers. ' A new objective has recently been introduced that restricts the incident infrared light to angles near grazing to the surface. ${ }^{2}$ At near-grazing incidence angles, the reflectance of the material is determined by the optical properties nearer to the surface than is the case for light incident closer to the perpendicular. The grazing angle reflectance spectrum can thus provide a vibrational analysis of the top few nanometers of the surface of the material.

The light path in such a grazing angle objective (GAO) is sketched in Fig. 1, which indicates that the infrared light is incident on the surface within a finite angular aperture. The polar angles of this aperture are nominally $65-85^{\circ}$ for the GAO considered here, so that the angle of incidence nearest to grazing is $85^{\circ}$. Light reflected from the sample is collected by the objective over the same angular aperture and conveyed to the infrared detector for acquisition of the (Fourier transformed) infrared reflectance spectrum.

The angular dependence of the intensity of the incident light within the objective's angular aperture is the main concern of this paper. Its importance is due to the source of the experimental reflectance; the reflected intensities

Received 19 August 1994; accepted 1 December 1994. at any given angle within the aperture are effectively summed up over the angular aperture to give an experimental value of the refiectance. At any given angle of incidence, these reflected intensities are determined by the product of the incident intensity at that angle and the reflection coefficient at that angle. These reflection coefficients are, of course, a strong function of the angle of incidence-going to one at grazing incidence and possibly much less than one at normal incidence. If the incident intensity is peaked at some particular angle of incidence, it is the reflectance at that particular angle that will be emphasized in the experimental reflectance. Therefore, understanding the experimental reflectance requires a knowledge of the dependence of the incident intensity on the angle of incidence within the objective's aperture. At present, information on the possible angular dependence of this intensity is not available.

In this paper, the GAO is characterized for quantitative spectroscopy with particular consideration of the dependence of the incident intensity with polar angle. Although a direct measurement of this angular dependence would be preferable, the less direct approach taken here is to test the validity of the simplest assumption: namely, that the incident intensity has no dependence on the polar angle and is effectively uniform within the angular aperture. In the section below, this test is done by comparing the experimental reflectance of a material with the reflectance calculated by mathematical integration over the objective's aperture. The materials chosen for this test are simple dielectrics with well-established indices of refraction in the infrared. Their angle-dependent reflectance can be calculated with the Fresnel reflection coefficients and integrated over the angular aperture, assuming no polar angle dependence of the incident intensity. Good agreement of experimental with calculated values would confirm the validity of the assumption. This supposition will be shown to be the case. In the following section, this approach to integrating over the angular aperture is then applied to the determination of the thickness of a thin liquid film on a metallic substrate. The characterization of the objective will illustrate the method for integrating over the angular aperture, and the application will illustrate the value of this approach to quantitative infrared reflection microspectroscopy.

\section{CHARACTERIZATION}

Theory. The calculation of the response of the GAO integrated over its angular aperture is essentially an ap- 
plication of the papers of Nicodemus on radiometry, and we refer the reader to them. ${ }^{3}$ At any angle $\theta$, the energy $E$ passed to the detector by the GAO is

$$
E(\nu)=\int I(\theta, \nu) R(\theta, \nu) \cos (\theta) \mathrm{d} \Omega
$$

where $I(\theta, \nu)$ is the energy incident on the surface at angle $\theta$ and frequency $\nu ; R(\theta, \nu)$ is the reflectance coefficient of the surface at angle $\theta$ and frequency $\nu$; and $\mathrm{d} \Omega=\sin \theta$ $\mathrm{d} \theta \mathrm{d} \phi$ is the differential solid angle at angle $\theta$. Assuming azimuthal symmetry and letting $x=\sin ^{2} \theta$,

$$
E(\nu)=\pi \int_{x_{\min }}^{x_{\max }} I(x, \nu) R(x, \nu) \mathrm{d} x
$$

where

$$
x_{\text {min }}=\sin ^{2}\left(65^{\circ}\right), \quad x_{\max }=\sin ^{2}\left(85^{\circ}\right)
$$

for the $65-85^{\circ}$ angular range of the GAO. The procedure in FT-IR microspectroscopy is to ratio a single-beam spectrum from a specimen to a single-beam "background" spectrum from a highly reflective surface, usually gold. With superscripts $s$ and $b$ used to denote specimen and background, respectively, the ratio $\rho$ is

$$
\begin{array}{r}
\rho(\nu)=\frac{\text { single-beam spectrum, specimen }}{\text { single-beam spectrum, background }} \\
\text { (experimental) } \\
=\frac{E^{\mathrm{s}}(\nu)}{E^{\mathrm{b}}(\nu)}=\frac{\int_{s_{\min }}^{s_{\max }} I(x, \nu) R^{\mathrm{s}}(x, \nu) \mathrm{d} x}{\int_{\imath_{\max }}^{x_{\max }} I(x, \nu) R^{\mathrm{b}}(x, \nu) \mathrm{d} x} \\
\text { (calculated). }
\end{array}
$$

It is now assumed that $I$ is not a function of $\theta$ or $\nu$, so

$$
\rho(\nu)=\frac{\int_{x_{\min }}^{x_{\max }} R^{\mathrm{s}}(x, \nu) \mathrm{d} x}{\int_{x_{\min }}^{x_{\max }} R^{\mathrm{b}}(x, \nu) \mathrm{d} x} .
$$

The absorbance $A$ is

$$
A(\nu)=\log _{10} \frac{1}{\rho(\nu)} .
$$

Here $\rho(v)$ is either an experimentally obtained spectrum (Eq. 4a) or a spectrum calculated (Eq. 4b) with the Fresnel formulas for reflectance.

This method is a sensitive test of the independence of the incident intensity on polar angle only if the surface examined exhibits a great variation in reflectance or absorbance over the angular range spanned by the GAO. If the reflectance or absorbance of the surface is constant over this angular range, as it essentially is for a clean metal surface, then the value of the integrated reflectance or absorbance will be independent of any structure in $I(\theta)$. Here, two surfaces, potassium bromide $(\mathrm{KBr})$ and silicon (Si), are used that are dielectric materials over the spectral range of the instrument, $6000-700 \mathrm{~cm} \mathrm{'}$. Their wellknown reflectances are shown in Fig. 2 . $\mathrm{KBr}$ exhibits a variation of 0.12 to 0.62 and $\mathrm{Si}$ of 0.32 to 0.59 for unpolarized light over the incident angle range of 65 to $85^{\circ}$. Note that, although the reflectance of both $s$ - and p-polarized light is monotonically increasing for $\mathrm{KBr}$, the reflectance of $p$-polarized light for Si exhibits a zero at its Brewster angle $\left(73.7^{\circ}\right)$ within the angular range. In a sense, the method is tested more severely with the use of silicon than it is with potassium bromide.

The reflectance of the surface of a semi-infinite solid may be calculated by first defining the following amplitudes of the propagation vectors of the electric field:

$$
\xi_{\mathrm{j}}=\sqrt{\epsilon_{\mathrm{j}}-\epsilon_{0} \sin ^{2} \theta}=\sqrt{\epsilon_{\mathrm{j}}-\epsilon_{0} x}
$$

where $\epsilon_{t}=\left(n_{j}+i k_{j}\right)^{2}$ is the frequency-dependent complex dielectric function of medium $j$ and $i=\sqrt{-1}$. $^{4}$ Here $n$ is the index of refraction and $k$ is the extinction coefficient. For the initial medium air, $\epsilon_{0}=1$.

For the dielectrics, $\epsilon_{1}$ is real and is given by:s

$$
\epsilon_{1}=1.52^{2}, \mathrm{KBr}
$$

and

$$
\epsilon_{1}=3.42^{2} \text {, Si. }
$$

The variation of the index of refraction of these materials over the infrared spectral range considered here was found to have a negligible effect on the results. The dielectric function for gold ${ }^{\text {th }}$ is complex:

$$
\epsilon_{1}=1-\frac{\nu_{p}^{2}}{\nu^{2}+i \nu \nu_{l}}, \mathrm{Au}
$$

with $v_{p}=72,500 \mathrm{~cm}{ }^{\prime}$ and $\nu_{t}=216 \mathrm{~cm} \quad$ '. The reflectances of $s$ - and $p$-polarized light are then given by

$$
\begin{aligned}
& R_{s}=\left|\frac{\xi_{0}-\xi_{1}}{\xi_{0}+\xi_{1}}\right|^{2} \\
& R_{p}=\left|\frac{\epsilon_{1} \xi_{0}-\epsilon_{0} \xi_{1}}{\epsilon_{1} \xi_{0}+\epsilon_{0} \xi_{1}}\right|^{2} .
\end{aligned}
$$

If the light incident on the sample is assumed to be unpolarized, then the reflectance $R$ is

$$
R=\frac{1}{2}\left(R_{s}+R_{p}\right) .
$$

It may be verified that these reflectances reduce to the well-known Fresnel reflection coefficients for the case of the dielectrics and were used to generate Fig. 2. This form for the reflectance, while not as common as that found in the usual texts, is quite general and allows the reflectance of an absorbing/reflecting medium with a complex dieletric function (such as gold) to be simply expressed and evaluated numerically. It also will allow the reflectance of a thin absorbing film on a gold substrate to be simply expressed.

These expressions for the reflectance were used in the numerical evaluation of the integral in Eq. 5 over the infrared spectral range.

Equipment. The instrument used here is a Nicolet NICPlan infrared microspectrometer equipped with a Spectra-Tech grazing angle objective. The MCT detector covers a spectral range of 6000 to $700 \mathrm{~cm}^{-1}$. The response of the detector at frequencies $200-400 \mathrm{~cm}^{-1}$ was verified to be $<2 \%$ of the maximum signal of a single-beam spec- 


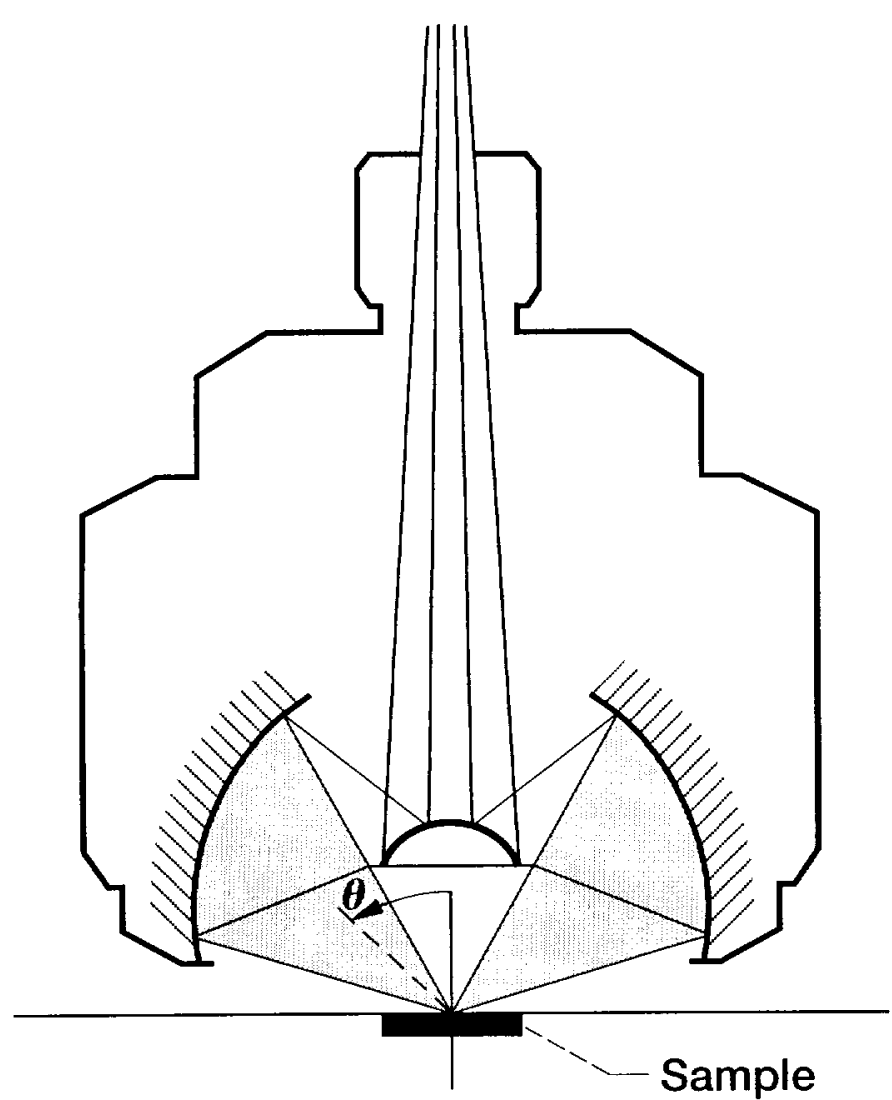

Frg. 1. Diagram of the grazing angle objective (GAO). The angular aperture is shaded.

trum. This is a requirement for the optimum linear response of the detector that is needed for the quantitative aspects considered here. The reflection spectra were acquired with an aperture restricting the circular region of analysis to $0.1 \mathrm{~mm}$. Spectral resolution was $8 \mathrm{~cm}^{-1}$. The number of coadded scans was 128 .

Reflectance spectra with unpolarized infrared light were acquired from $\mathrm{KBr}$ and $\mathrm{Si}$ polished infrared windows that were $2 \mathrm{~mm}$ thick. The background reflectance spectrum was acquired from a silicon window coated with $150 \mathrm{~nm}$ of gold over $15 \mathrm{~nm}$ of chromium.

In addition to the infrared light reflected from the top surface, some infrared light is also refracted in to the transparent windows, reflected from the bottom surface, and emerges from the top surface displaced laterally from the entry point. This emergent light might contribute to the reflected intensity. However, the same results were obtained for windows with bottom surfaces gold-plated, which gives near-total reflectance from this bottom interface and greatly increases the intensity of the emergent light. Therefore this emergent light is not collected and must emerge from the top surface outside the area to which the objective is sensitive. The collected intensity is thus due only to top-surface reflection, which is calculated as indicated above.

Results. The results for reflectance from $\mathrm{KBr}$ and $\mathrm{Si}$ are presented in Fig. 3 for the frequency range 6000 to $700 \mathrm{~cm}$ '. The calculated specular reflectance values over the $65-85^{\circ}$ range spanned by GAO, taken from Fig. 2, are indicated by the vertical bars. These specular reflec-
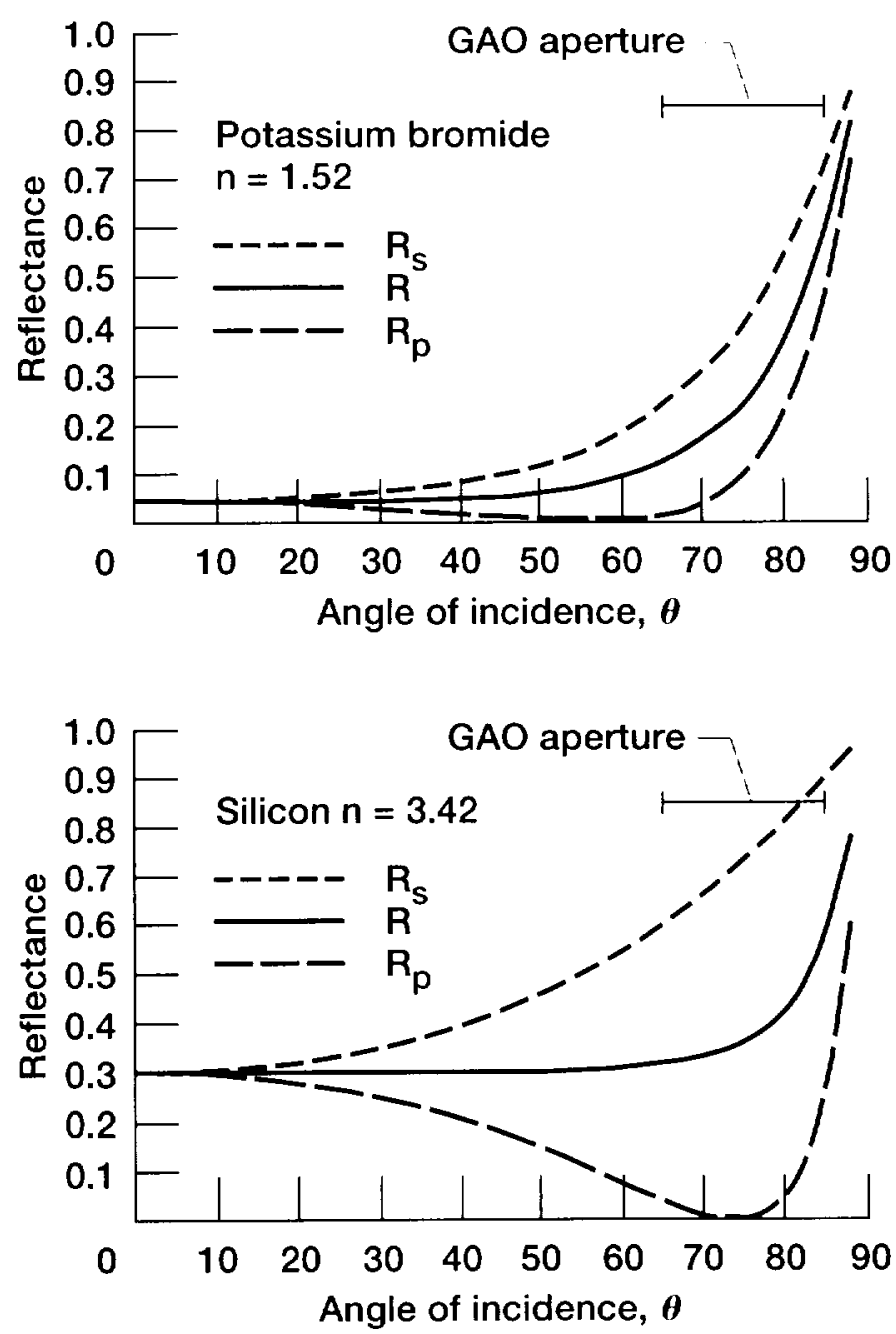

Fig. 2. Reflectance of potassium bromide and silicon in the infrared vs. angle of incidence, $\theta$. The angular aperture of the GAO is indicated by the horizontal bar.

tances are integrated over the angular aperture to yield an integrated reflectance at a given frequency. Since the index of refraction of $\mathrm{KBr}$ and $\mathrm{Si}$ and the reflectance of gold do not vary appreciably across this frequency range, the calculated values are essentially the same for all fre-

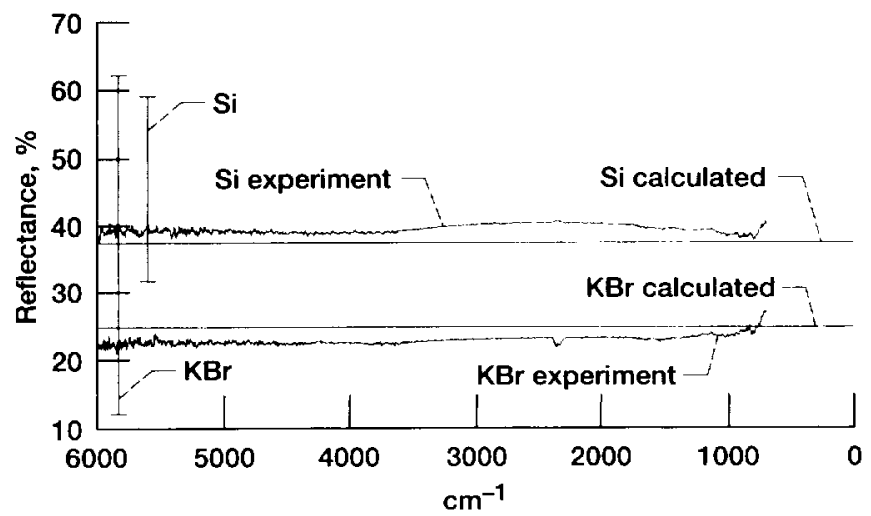

FIG. 3. Spectral reflectance of $\mathrm{KBr}$ and Si with the GAO. The specular reflectance over the angular range of the GAO is indicated by the vertical bars. 
quencies and are indicated by the horizontal solid lines. The specular reflectance of $\mathrm{KBr}$ and $\mathrm{Si}$ integrate to calculated reflectances of $25 \%$ and $37.2 \%$, respectively.

The experimental reflectance spectra in Fig. 3 are generally quite flat except for a small increase at the lowfrequency end of the spectrum. For $\mathrm{KBr}$, the experimental value of $\sim 22.8 \%$ is somewhat $(\sim 9 \%)$ less than the calculated value of $25 \%$, while for Si the experimental value of $39.5 \%$ is somewhat $(\sim 7 \%)$ higher than the calculated value of $37.2 \%$. Thus the experimental values are within $\sim 2.5$ reflectance percentage points of the values calculated under the assumption of no polar angle dependence of the incident intensity.

The calculated reflectance depends on the limits of integration over the objective's angular aperture. Even though the manufacturer gives these limits as $65-85^{\circ}$, departures from these nominal values are conceivable. Calculations were thus performed to test the sensitivity of the reflectance to the value of the endpoint angles of the GAO. The integrated reflectance is more sensitive to these limits for the case of $\mathrm{KBr}$ than for $\mathrm{Si}$. This behavior is due to the greater variation of the $\mathrm{KBr}$ reflectance over these angles, as indicated in Fig. 2. For $\mathrm{KBr}$, the reflectance is least sensitive to the angular limits $(\sim 0.2 \%$ per degree) for a symmetrical expansion or contraction of the angular range around the $75^{\circ}$ midpoint. A uniform translation of the $20^{\circ}$ angular range to higher or lower values results in a change of $\sim 1.6 \%$ per degree, while an asymmetrical expansion or contraction changes the reflectance by $\sim 1 \%$ per degree. This sensitivity of the calculated reflectance offers the opportunity of choosing values of the angular limits that brings the calculated reflectance for $\mathrm{KBr}$ down into agreement with the measured value. However, this approach will only enhance the disagreement for the Si case, since its calculated reflectance will be reduced even further below the measured value. In any case, as discussed above, there are factors other than the angular limits that may account for the relatively small differences between calculated and measured reflectance values. It is thus considered best at this point to accept the values for the limits of the angular aperture as stated by the manufacturer.

Discussion. In this section, a comparison between calculation and experiment is made to test the assumption of angular independence of incident intensity. However, this comparison is a valid test of the assumption only if the reflectances of the test surfaces used here $(\mathrm{Si}, \mathrm{KBr}$, and $\mathrm{Au}$ ) actually obey specular reflection Fresnel theory. These materials are chemically pure, so that their infrared optical constants taken from the literature are considered valid. Here, any deviation from specular reflection Fresnel theory is considered to be due to light scattering from defects such as internal voids or surface roughness. Since no independent assessment of the specularity of the test surfaces is available here, only speculation on this issue is possible. Best specularity is assigned to the $\mathrm{Si}$, a highly polished window manufactured for infrared optical application, with no surface topography apparent to the eye at $100 \times$. The Au film sputter-deposited on the Si window was as apparently specular as its substrate. However, the $\mathrm{KBr}$ crystal did exhibit some surface roughness apparent at $100 \times$ magnification. On this basis, higher confidence in the calculated values is assigned to the $\mathrm{Si} / \mathrm{Au}$ reflectance ratio. The $\mathrm{KBr}$ reflectance ratio may be subject to some light scattering that would lower the measured value below the calculated value.

With these factors in mind, the degree of agreement in Fig. 3 between the measured values and the values calculated under the assumption of angular independence of the incident light can be assessed. The experimental values of $\mathrm{Si} / \mathrm{Au}$, in which there is greatest confidence in fulfilling the conditions of the test, is seen to be only about two reflectance percentage points above the calculated values. This is good agreement. It is important to note, however, that the degree of agreement found here cannot be taken for granted; initially, the measured reflectance was $\sim 50 \%$, greatly exceeding the calculated value. Our objective turned out to have a misaligned mirror, subsequently corrected by the manufacturer to yield the values reported here. Thus the degree of agreement of measured with calculated values for $\mathrm{Si}$ appears to be a useful field test for the alignment of the objective's mirrors.* It is also noted that a measured reflectance of $38.6 \%$, even closer to the calculated $37.2 \%$, was obtained with another instrument† of the same manufacturer and thus possibly better aligned than our instrument.

The experimental values for $\mathrm{KBr} / \mathrm{Au}$ were lower than the calculated values by about two reflectance percentage points. The lower values are consistent with the possible scattering of light by the surface roughness. However, this discrepancy may well be due to other factors, such as residual optical misalignment of the instrument. It is also interesting to note the degree of agreement between calculated and measured values achieved without the necessity of invoking partial polarization of the incident light. This observation is somewhat surprising since the many reflections undergone by the light prior to reflection from the sample may be expected to result in its partial polarization. Thus, the agreement between calculated and measured values is impressively close.

The agreement between calculated and measured spectral reflectances provides support for the hypothesis that the incident radiation intensity is independent of polar angle. This agreement, however, does not constitute a proof, since there may be an angular dependence that, upon integration weighted by the reflectance, results in the same degree of agreement. Thus the conclusion here is that the incident radiation intensity may be considered uniform for calculational purposes, but a determination of the true angular dependence awaits a direct measurement.

Finally, the use of an "effective" angle of incidence can be considered. An effective angle of $75^{\circ}$, the midpoint of the angular range of the objective, can be used in a calculation of specular reflection without the exact integration over the full angular aperture. In fact, such an approach was found to give quite close agreement to the fully integrated values in many cases. This approach should be used with caution, however, since it manifestly fails for $p$-polarized light on $\mathrm{Si}$. In this case, $75^{\circ}$ is quite

\footnotetext{
* This sensitivity to alignment was not apparent for $\mathrm{KBr}$ and seems to be the case only for those materials whose Brewster's angle is within the angular range spanned by the objective.

$\dagger$ The cooperation of Duane Smith of the Timken Company, Canton, Ohio, is gratefully acknowledged.
} 
close to the $\mathrm{Si}$ Brewster's angle of $73.7^{\circ}$ at which the p-reflectance is zero. As Fig. 2 shows, the values of the $p$-reflectance over the angular range are much higher than at the midpoint, so the properly integrated reflectance will be much higher than the midpoint value. Therefore, if optical constants of a material are at all available to perform a calculation, the full integration may as well be used, without the uncertainty associated with using an effective angle.

\section{APPLICATION}

Theory. The approach to quantitative reflection microspectroscopy developed above is now applied to the determination of the thickness of a liquid film on a gold substrate. This is done by comparing the film's measured absorbance to the thickness-dependent absorbance calculated from thin-film Fresnel reflectance theory integrated over the angular aperture. The liquid used here is Krytox 16256, one of the perfluoropolyethers (PFPEs) used as both diffusion pump oils and lubricants for mechanisms in vacuum. ${ }^{7}$ The chemical structure of this liquid is

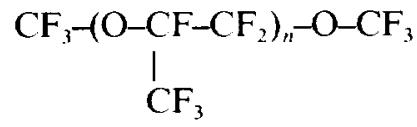

where $n \cong 65$ for a molecular weight of $\sim 11,000$.

The optical properties of a number of PFPEs have been studied by Pacansky et al., and the optical dielectric function $\epsilon(\nu)$ in the infrared for a PFPE of the Krytox type has been provided as a convenient oscillator model by Novotny et al. ${ }^{8,9}$

The calculation of the reflectance of this system uses the quantities $\xi_{j}$ defined above, where $\xi_{0}$ refers to air, $\xi_{2}$ refers to gold, and $\xi_{1}$ now refers to the liquid

$$
\xi_{1}(v)=\sqrt{\epsilon_{1}(v)-\epsilon_{0} x}
$$

where

$$
\epsilon_{1}(\nu)=\epsilon_{\infty}+\sum_{j=1}^{n} \frac{\nu_{j}^{2} f_{j}}{\nu_{j}^{2}-\nu^{2}-i \nu \gamma_{j}} .
$$

Here $\epsilon_{x}=1.7$, the square of the index of refraction in the visible, and the values of the oscillator parameters $\nu_{j}, f_{j}$, and $\gamma_{i}$ are listed in Table I, from Novotny et al. ${ }^{9}$

The reflectance amplitude $r_{1}$ for the air/film interface and $r_{2}$ for the film/substrate interface is

$$
r_{1 s}=\frac{\xi_{0}-\xi_{1}}{\xi_{0}+\xi_{1}}, \quad r_{2 s}=\frac{\xi_{1}-\xi_{2}}{\xi_{1}+\xi_{2}}
$$

for $s$-polarized light and

$$
r_{1 p}=\frac{\epsilon_{1} \xi_{0}-\epsilon_{0} \xi_{1}}{\epsilon_{1} \xi_{0}+\epsilon_{0} \xi_{1}}, \quad r_{2 p}=\frac{\epsilon_{2} \xi_{1}-\epsilon_{1} \xi_{2}}{\epsilon_{2} \xi_{1}+\epsilon_{1} \xi_{2}}
$$

for $p$-polarized light.

Then the reflectance of this system with film thickness $d$ for $s$-polarized light is

$$
R_{s}=\left|\frac{r_{1 s}+r_{2 s} e^{4 \pi v v d \xi_{1}}}{1+r_{1, s} r_{2 s} e^{4 \pi v i d \xi_{i}}}\right|^{2}
$$

with a similar expression, $s \rightarrow p$, for $p$-polarized light. For unpolarized light, Eq. 10 holds. Integrated reflectance
TABLE I. Main infrared absorption bands of Krytox. The $f_{i}$ and $\gamma_{i}$ are oscillator strengths and widths of vibrational bands at $\nu_{i}$.

\begin{tabular}{cccc}
\hline$i$ & $\nu_{i}\left(\mathrm{~cm}^{1}\right)$ & $f_{i}$ & $\gamma_{i}\left(\mathrm{~cm}^{1}\right)$ \\
\hline 9 & 740 & 0.013 & 25 \\
8 & 805 & 0.008 & 30 \\
7 & 980 & 0.024 & 20 \\
6 & 995 & 0.006 & 20 \\
5 & 1060 & 0.008 & 30 \\
4 & 1125 & 0.055 & 50 \\
3 & 1180 & 0.025 & 50 \\
2 & 1240 & 0.081 & 50 \\
1 & 1305 & 0.0076 & 20 \\
\hline
\end{tabular}

spectra were calculated with Eq. 5 and displayed as absorption spectra with Eq. 6 .

Experiment. The liquid film was applied to the goldcoated silicon substrate by dipcoating from a solution of $3 \mathrm{~g}$ Krytox in $100 \mathrm{~mL}$ of Vertrelł at room temperature. The film thickness was determined to be $47.4 \mathrm{~nm}$ by variable angle $\left(65^{\circ}, 70^{\circ}, 75^{\circ}\right)$ spectroscopic ellipsometry in the visible $(300-800 \mathrm{~nm})$.

Infrared reflectance spectra were acquired at a resolution of $2 \mathrm{~cm}^{-1}$ for both the film-covered substrate and the gold background. The number of coadded scans for a spectrum was 400 .

Results. Calculated spectra are presented first, and then the experimental spectrum is presented. A comparison of the calculated and experimental spectra can then determine the film thickness via the infrared spectrum. The film thickness determined this way is then compared with the film thickness determined by spectroscopic ellipsometry in the visible to evaluate the capabilities of this approach to quantitative infrared spectroscopy.

The calculated spectra for two thicknesses are shown in the inset in Fig. 4. The maximum absorbance of such spectra is shown as a function of thickness in this figure. The spectrum shape is invariant for $d<100 \mathrm{~nm}$ with only the amplitude changing. Spectral shapes for $d>100$ $\mathrm{nm}$ start to change because of the appearance of the "transverse optical mode" in the spectra of thicker films, and this behavior has been discussed by Yen and Wong. ${ }^{10}$ Another way of looking at this pattern is that the spectra of thicker films start to resemble the spectra of semiinfinite Krytox, without contributions of multiple reflections from the gold substrate. The plot of maximum absorbance vs. thickness provided a "calibration curve", whereby the maximum absorbance of an experimental spectrum can be used to obtain a film thickness.

Absorbance spectra of the Krytox film were obtained from three different points near the center of the sample. The shape of the spectra were the same, with only the amplitude differing. Their maximum absorbances were $0.09,0.108$, and 0.111 , corresponding to film thicknesses of $46,57.5$, and $59.5 \mathrm{~nm}$. One of these spectra is shown in Fig. 5 , together with a spectrum calculated with $d=$ $59.5 \mathrm{~nm}$. This figure illustrates the procedure for obtaining a film thickness from an experimental spectrum by finding the thickness for which the calculated spectrum has the same maximum absorbance as the experimental spectrum.

Discussion. The experimental spectra exhibit some variability in their maximum absorbance that may be

$\ddagger$ Vertrel 245 a cyclic perfluorocarbon available from Dupont. 


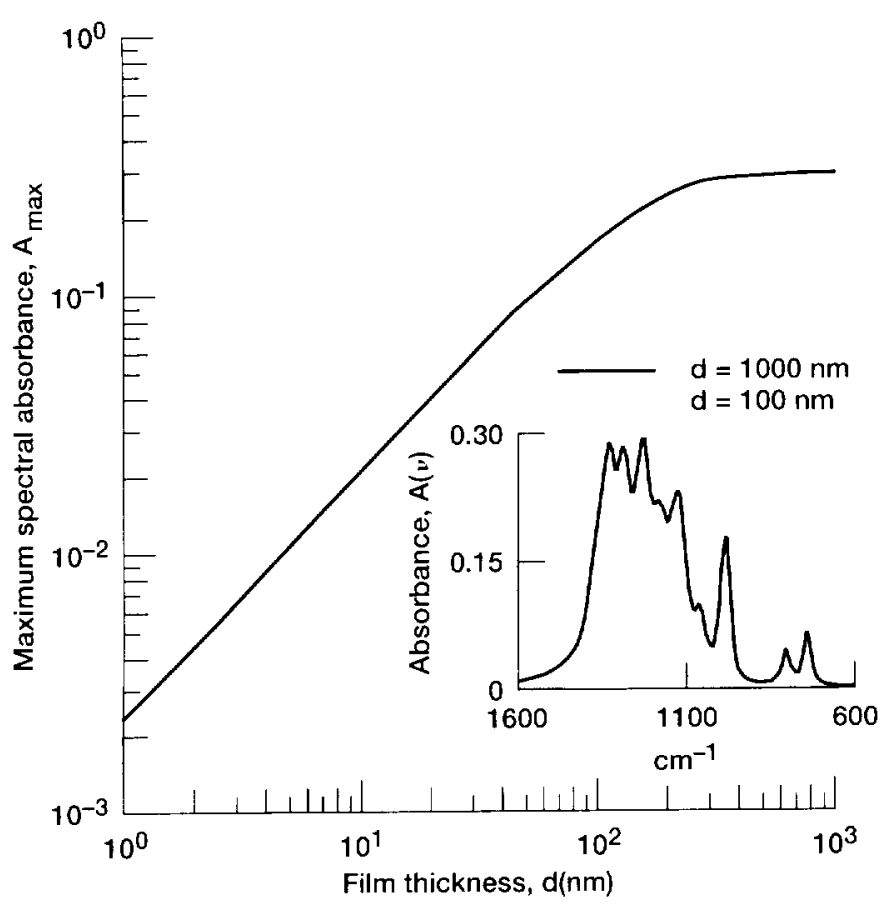

FIG. 4. Calculated maximum spectral absorbance of Krytox film on gold vs. film thickness. Inset: calculated absorbance spectra for film thicknesses of 100 and $1000 \mathrm{~nm}$

due to true lateral variation in the thickness of this dipcoated film. Recall that the spectra were obtained from regions $\sim 0.1 \mathrm{~mm}$ in diameter. However, the thickness of the film determined by ellipsometry probes a surface region $\sim 2 \mathrm{~mm} \times 6 \mathrm{~mm}$. This region is due to the size of the incident monochromatic light beam and its spread by oblique incidence. Thus the thickness determined ellipsometrically, $47.4 \mathrm{~nm}$, may be considered to average over any thickness variations that exist on the millimeter scale. It is this factor that may account for the variability in the spectral amplitude as well as the difference in thickness determined by the two methods.

Another source of uncertainty that may account for the differences is the applicability of the published infrared optical constants of a fluid generally of the Krytox type ${ }^{8}$ and its oscillator model ${ }^{9}$ (Table I) to the particular Krytox fluid used here. Krytox is available in a range of molecular weights, and type 16256 is on the high end of the range. It may be that the published values do not adequately describe type 16256, and this difference may account for discrepancies in film thickness determination. This applicability may be assessed by inspecting the agreement of the spectra in Fig. 5 with each other. On the positive side, the overall spectral shape and positions of the major maxima are in good agreement. On the other hand, the calculated width of the major features at $\sim 1300 \mathrm{~cm}^{-1}$ is larger than the experimental value. This observation implies that the $\gamma_{1}$ and $\gamma_{2}$ of the oscillator model may be too large. In addition, matching the experimental and calculated maximum absorbances does not yield very good agreement for the amplitudes of the features between 1100 and $1200 \mathrm{~cm}^{-1}$. Thus there may be some error in the relative oscillator strengths $\left(f_{i}\right)$ in Table I. Finally, the bands at 740 and $805 \mathrm{~cm}$ ' of the oscillator model do not appear in the experimental spectra, although this sit-

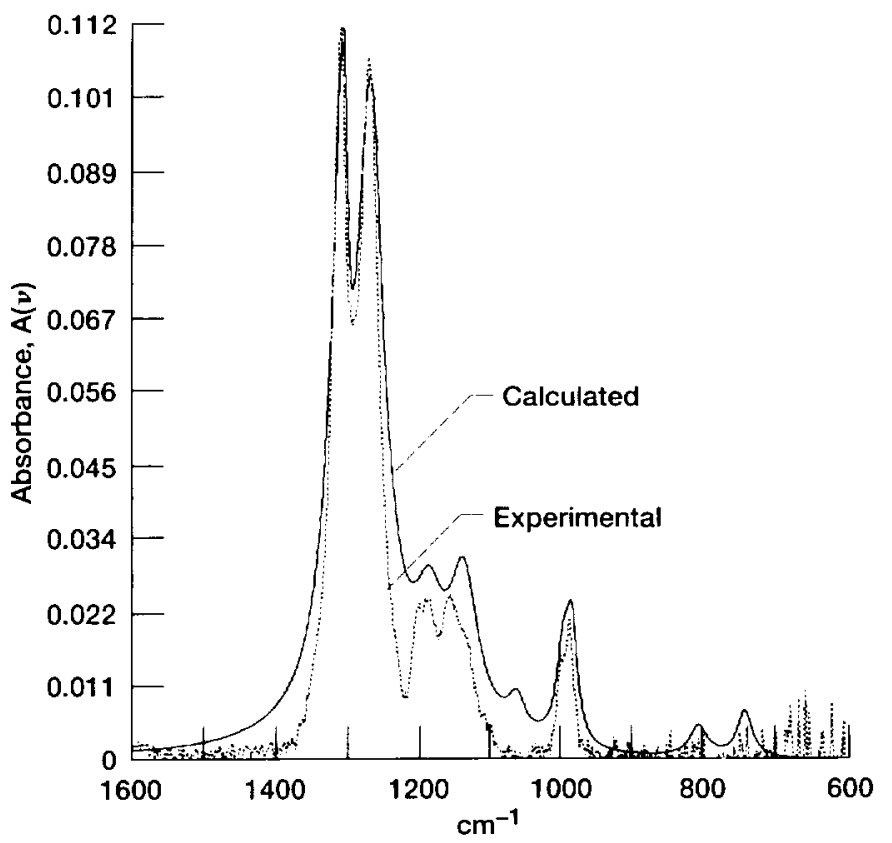

Fig. 5. Absorbance spectra for Krytox films on gold. Dotted line: experimental spectrum for dipcoated Krytox 16256 . Solid line: calculated spectrum with the use of oscillator parameters of Table I and $d=$ $59.5 \mathrm{~nm}$.

uation may simply be due to a lack of adequate signalto-noise ratio, rather than a true absence of these bands.

Thus, even though the oscillator model describes the major features of the experimental spectra reasonably well, these differences indicated the limitation of the model for the quantitative determination of film thickness. A redetermination of the infrared optical constants for this particular fluid may resolve these differences and allow even closer agreement between experimental and calculated spectra.

The validation of the approach to quantitative infrared microspectroscopy depends on two elements: (1) the homogeneity of the dipcoated film to allow the area-averaged, ellipsometrically determined thickness to be compared with the spectroscopically determined thickness at a local area, and (2) the availability of infrared optical constants that are valid for the material being examined. As discussed above, neither of these criteria may be satisfied totally in this case. The latter criterion is regarded as the most serious limitation to the general application of the approach discussed here. Although there is a compilation of the infrared optical constants for a limited number of materials, ${ }^{5}$ the information for liquids is generally not available except for some specific cases." In fact, the experimental techniques to obtain reliable infrared optical constants of any materials are still a matter of current discussion. ${ }^{12}$ It is hoped that future activities will lead to the availability of this information for a wide range of materials, especially liquids, and thus allow the more general application of the approach to quantitative infrared microspectroscopy described here.

\section{CONCLUSION}

The grazing angle objective for an infrared microspectrometer has been studied to determine if there is a sig- 
nificant nonuniformity in the angular distribution of light incident on the surface being examined. A comparison of the reflectance calculated by Fresnel reflection theory to the experimental reflectance of potassium bromide and silicon yielded fairly good agreement, indicating that the objective can be treated as if this intensity of the incident light exhibits no angular variation within the angular aperture of the objective. Therefore, this paper has illustrated a method for more quantitative use of a grazing angle objective for infrared reflectance microspectroscopy.

The technique of integration of the Fresnel specular reflectance over the angular aperture was applied to the determination of the thickness of a thin liquid film on a gold substrate. This thickness was in fair agreement with that obtained by variable-angle spectroscopic ellipsometry (VASE) in the visible. The usefulness of this technique for quantitative infrared microspectroscopy requires the availability of infrared optical constants of the material being examined. Although this information is not available for many materials, future developments may remedy this limitation.

\section{ACKNOWLEDGMENTS}

Dr. Samuel Alterovitz and Dr. Alan Heyd performed the fluid film thickness measurements by variable-angle spectroscopic ellipsometry. Critical comments by Dr. John Reffner are appreciated.
1. J. E. Katon, A. J. Sommer, and P. L. Lang, Appl. Spectrosc. Rev. 25, 173 (1989).

2. Patent $\# 4,810,077$, Spectra-Tech Inc. Stanford, Connecticut. See also J. A. Reffner, W. T. Wihlborg, and S. W. Strand, Am. Lab. April (1991).

3. F. W. Nicodemus, Am. J. Phys. 31, 386 (1963); idem, Appl. Opt. 4, 767 (1965).

4. S. V. Pepper, J. Opt. Soc. Am. 60, 805 (1970).

5. E. D. Palik, "Potassium Bromide", in Handbook of Optical Constants of Solids II, E. D. Palik, Ed. (Academic Press, New York, 1991), p. 989; D. F. Edwards, "Silicon", in Handbook of Optical Constants of Solids, E. D. Palik, Ed. (Academic Press, New York, 1985), p. 547.

6. M. A. Ordal, L. L. Long, R. S. Bell, S. E. Bell, R. R. Bell, R. W. Alexander, Jr., and C. A. Ward, Appl. Opt. 22, 1099 (1983).

7. T. W. Del Pesco, "Perfluoroalkylpolyethers", in Synthetic Lubricants and High Performance Functional Fluids, R. L. Shubkin, Ed. (Marcel Dekker, New York, 1993), Chap. 6, p. 145.

8. J. Pacansky, C. D. England, and W. Waltman, Appl. Spectrosc. 40, 8 (1986); J. Pacansky, R. J. Waltman, and M. Maier, J. Phys. Chem. 91, 1225 (1987)

9. V. J. Novotny, I. Hussla, J.-M. Turlet, and M. R. Philpot, J. Chem. Phys. 90, 5861 (1989).

10. Y.-S. Yen and J. S. Wong, J. Phys. Chem. 93, 7208 (1989).

11. J. P. Hawranek and R. N. Jones, Spectrochim. Acta 32A, 111 (1976).

12. J. B. Huang and M. W. Urban, Appl. Spectrosc. 46, 1666 (1992). 